\title{
Experimental and numerical study of cemented bone-implant interface behaviour
}

\author{
D. Kytyr, T. Doktor \\ Czech Technical University in Prague, Faculty of Transportation Sciences, Department of Mechanics and Materials, Na Florenci \\ 25, 11000 Prague 1 \\ xkytyn@fd.cvut.cz,xdok.tor@fd.cvut.cr.
}

O. Jirousek, P. Zlamal

Academy of Sciences of the Czech Republic, Institute of Theoretical and Applied Mechanics, Prosecka' 76, 19000

Prague 9

jirousek@itam.cas.cv,zlamal@itam.cas.cz.

D. Pokorny

Charles University, Faculty of medicine, Orthopaedic Clinic, V Uvalu 84, 15006 Prague 5

david.pokorny@lf1.cuni.cz.

\begin{abstract}
Although the total hip replacement (THR) is a long-proven method of surgical treatment of diseases and disorders of the human hip, the surgery brings some risk of long-term instability of the joint. The aim of the research was to investigate the cemented bone-implant interface behavior. The main problems (cement layer degradation and bone-cement interface debonding) during physiological loading conditions have been investigated using a custom hip simulator. The experimental setup was designed to allow cyclic loading of the sample of pelvic bone with implanted cemented acetabular component. The hip contact force of required direction and magnitude was applied to the implant using a spherical femoral component head. The most unfavorable activity (downstairs walking) was simulated. The process of damage accumulation in the fixation was monitored by repeated scanning using high resolution micro Computed Tomography $(\mu \mathrm{CT})$. Use of microfocus source and large high-resolution flat panel detector allows investigation of structural changes and crack propagation both in the cement layer and the trabecular bone.
\end{abstract}

KEYWORDS. Bone-cement interface; Hip simulator; $\mu \mathrm{CT}$; Crack detection.

\section{INTRODUCTION}

7 otal hip arthroplasty or surgical replacement of the hip joint with an artificial prosthesis is a reconstructive procedure that has improved the management of those diseases of the hip joint that have responded poorly to conventional medical therapy. Success of the treatment can be disturbed by several causes, besides the insufficient 
rehabilitation or rejection of replacement, aseptic loosening is one of the most frequent reasons of failure of the THR. The role of failure in aseptic loosening of cemented total hip replacements has been extensively studied mainly in femoral components. The process of the cement mantle degradation and bone-cement interface debonding of acetabular component was not yet satisfactorily described. The rate of acetabular component failure is more then two times higher than femoral one. The most significant work in this field include research of Zant et al. [2,3] at University of Portsmouth. The bone-cement interface has been studied using mechanical testing and numerical analyses, however, to our knowledge, there is no study on using $\mu \mathrm{CT}$ to observe the structural changes in the cement layer and trabecular bone. The aim of the research was to investigate the degradation caused by the cyclic mechanical loading using radiological methods. Visualisation of the trabecular bone structure and the cement layer changes provided detailed information about implant instability progress.

\section{MATERIALS AND METHODS}

\section{Bone specimen preparation}

7 he experiments and measurements were carried out using wet anatomical specimen of human hemipelvic bone. The specimen was preserved by immersion in $4 \%$ phosphate $\left(\mathrm{Na}_{2} \mathrm{HPO}_{4}\right)$ buffered paraformaldehyde $\left(\mathrm{OH}\left(\mathrm{CH}_{2} \mathrm{O}\right) \mathrm{nH}\right)$. With regards to the size of the used X-ray detector it was necessary to slightly adjust the dimensions of the specimen to fit the detector. The bone was resected in its ischial, pubic and iliac part; the acetabular area remained intact. The pelvis was scanned using a custom microtomography device prior any mechanical testing. After the scanning of the intact acetabular region, cemented acetabular component was implanted.

Cemented acetabular cup Poldi (Beznoska s.r.o.) was used. For proper functioning of the cup and its fixation it is essential to keep the thickness of the cement layer constant through the whole acetabular region. A good compression into the bone bed and the notches on the surface of the cup constrains the movements of the implant. The outer shape of the cup is a spherical surface with a transverse radial notch located along the perimeter of the implant. The implant was made from a modified highly crosslinked ultra-high-molecular-weight polyethylene (UHMWPE) developed by the Institute of Macromolecular Chemistry of the Czech Academy of Sciences (Patent No. 297700).

Polymethylmethacrylate (PMMA) bone cement $\left(\left(\mathrm{C}_{5} \mathrm{O}_{2} \mathrm{H}_{8}\right) \mathrm{n}\right.$ methacrylic acid methyl ester), produced by mixing the powdery substance and liquid components was used for implant fixation. Acetabular component implantation procedure is depicted in Fig. 1.
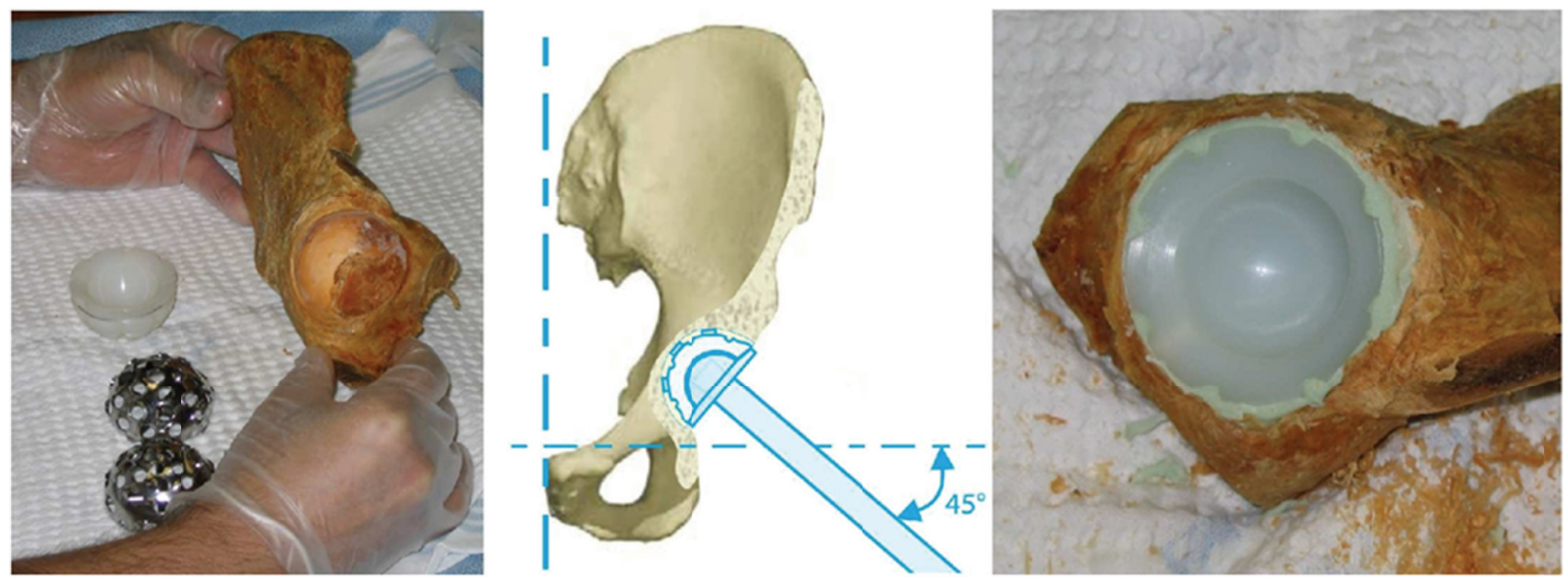

Figure 1: Acetabular component implantation

\section{Loading test}

A new hip joint simulator was developed for acetabular implant testing (see Fig. 2). The experimental setup was designed to allow cyclic loading of the pelvic bone sample with implanted cemented acetabular component. For the cyclic loading servo-hydraulic Instron 1603 was used. The outputs of the force and displacement transducers were captured with $10 \mathrm{~Hz}$ sampling frequency. A sinusoidal force with a given amplitude was applied. The hip contact force of required direction 
and magnitude was applied to the implant using spherical femoral component head. According to the hip contact forces measurement [1] mean value $(1400 \mathrm{~N})$ and amplitude $(1000 \mathrm{~N})$ were chosen to simulate the most unfavorable activity (downstairs walking) with $4 \mathrm{~Hz}$ frequency. This frequency is higher than that of normal walking but without any effect to implant durability [3]. The pelvic bone was supported by an epoxy mould. During the test, the specimen was moistened by saline solution.

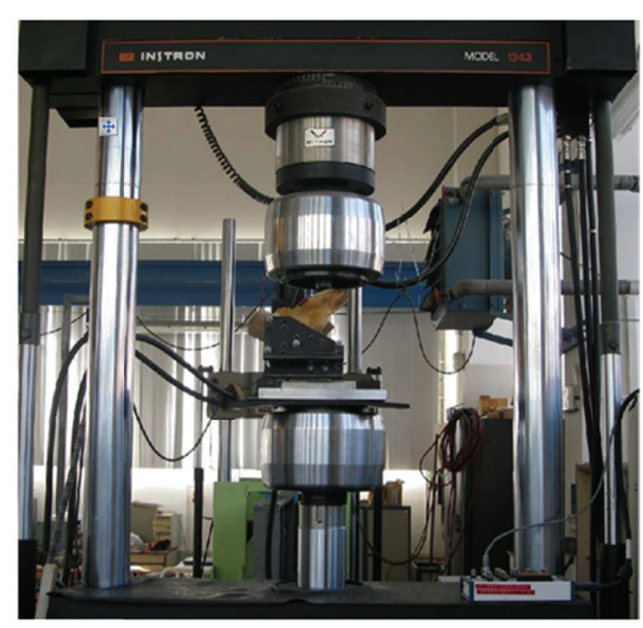

Figure 2: The hip simulator.

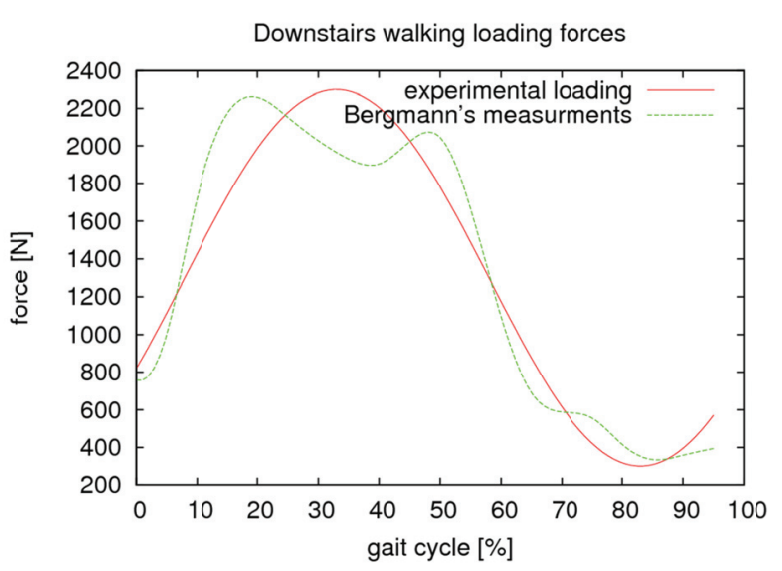

Figure 3: Loading cycle.

\section{Material testing}

Knowledge of material properties is a prerequisite for performing mechanical analysis, especially in case of numerical simulations. There is very wide range of trabecular bone material properties $(\mathrm{E}=0.1-4.5 \mathrm{GPa})$ reported in literature almost no correlation between the Young's modulus and anatomical location. The high discrepancy in the published material properties was the reason for experimental assessment of material properties. Material properties of trabecular bone can be assessed by mechanical testing, but in this case strain measurement is challenging due to the brittle nature of the samples [4]. To measure the strain using standard procedures i.e. strain gauge (difficult attachment to the sample surface) nor extensometer (possible damage of the sample surface) is inconvenient.

The pulse transmission ultrasonic technique [5] is a quick non-destructive method for Young's modulus estimation. A good agreement between the ultrasonically determined and mechanically measured elastic moduli of the cancellous bone structure have been reported [6]. A series of 20 samples from 5 different donors was tested. The specimens of $5 \mathrm{~mm}$ diameter and $20-30 \mathrm{~mm}$ length were drilled out from femoral heads and delipidated. The experiment depisted in Fig. 4 was carried out using USG 20 (Krompholz Geotron Elektronik, FRG) used with a $250 \mathrm{kHz}$ vibrator (USG -T) and receiver (USE-T). The velocity of the longitudinal ultrasonic waves' propagation depends on the mechanical properties of the material, therefore the Young's modulus can be obtained from the formula (1) coupling elasticity modulus $\mathrm{E}_{\text {ad, }}$, wave velocity $\mathrm{v}$ and bone density $\varrho$, (for details see e.g. [7]).

$$
E_{a d}=v^{2} \rho
$$

Density of pure trabecular bone was obtained by sample weighting and volume measurements. Ultrasound probe was used to measure the time of wave propagation. For simplification the path of the wave propagation was taken equal to the sample length. Then formula for the elastic modulus can be expressed as:

$$
E_{a d}=\frac{l^{2} m}{t^{2} v}
$$

The kind of measurement yields the so called adiabatic (or dynamical) Young's modulus which is larger than static (or isothermic) modulus obtained from tensile or compressive tests. Relationship between the adiabatic $\mathrm{E}_{\mathrm{ad}}$ and isothermic $\mathrm{E}_{\text {iso }}$ moduli depends on the coefficient of thermal expansion $\alpha$ and the specific heat of the material $C_{p}$ at constant stress 
and absolute temperature $\mathrm{T}$ :

$$
\frac{E_{a d}}{E_{\text {iso }}}=1+\alpha^{2} T \frac{E_{a d}}{\rho C_{p}}
$$

From the set of samples the value of elastic modulus $(E=853 \pm 72 \mathrm{MPa})$ was obtained. This value fits in the range of reported elastic moduli and was used in numerical analysis.

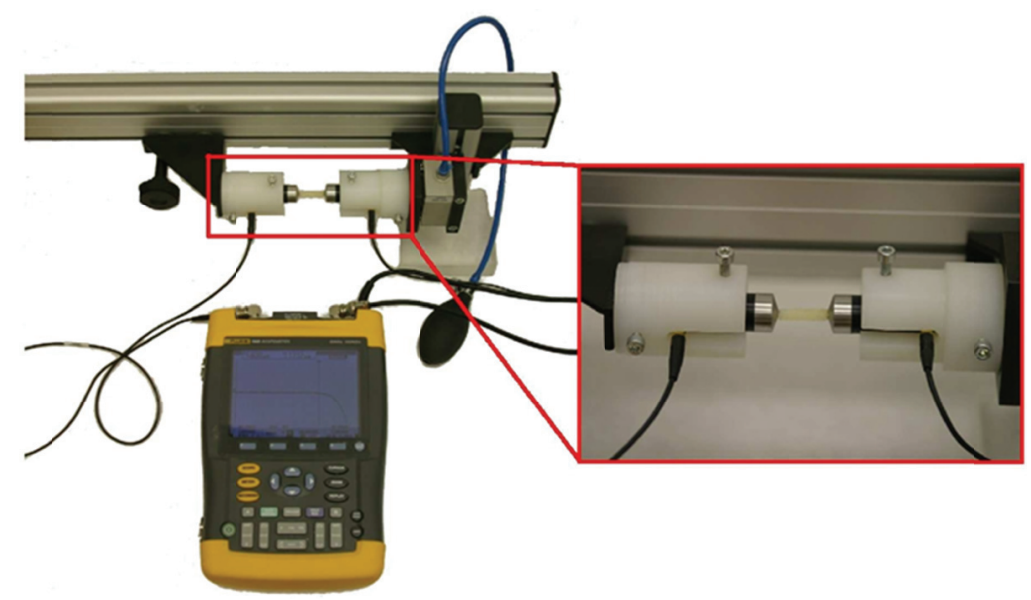

Figure 4: Ultrasonic measurement of elastic modulus.

\section{Numerical analysis}

The primary purpose of the numerical analysis was the assessment of the degradation of acetabular replacement fixation and comparison with its behavior during the loading tests. Detailed three-dimensional finite element model of the acetabular cup and the cement mantle implanted in a small bone block was developed. The analysis of the gait divided in 20 loadsteps ascertained stress field distribution at the bone-cement interface during gait cycle.

A solid model was created in parametric modeller Inventor (Autodesk, Inc.). The model was composed of 4 separately designed components. The geometry of the acetabular implant was obtained from datasheet provided by the manufacturer (Beznoska s.r.o.). Inner diameter was $32 \mathrm{~mm}$, outer $49 \mathrm{~mm}$. The geometry of femoral component was simplied to include the spherical head $(\oslash \mathrm{D}=32 \mathrm{~mm})$ and the cylindrical neck. The cement mantle was designed by embossing the cup to the hemisphere. The thickness of this layer was $3 \mathrm{~mm}$. The bone was modeled regardless of the true geometry as a cylindrical socket. The implant was surrounded with minimally $15 \mathrm{~mm}$ of the bone material in all directions. Final assembly (parts and constrains) was exported in the STEP format.

HyperMesh (Altair Engineering, Inc.) finite element pre-processor was used for model discretization. The goemetry in the STEP format is defined by areas, therefore the surface was discretized by quadratic triangular elements first. Because of the thin geometry and small radius of the cement mantle quadratic elements were chosen. Volume mesh of 10-node tetrahedral elements shown in Fig. 5 was generated with respect to surface mesh. Each component mesh was exported separately using the same coordinate system. HyperMesh native format was converted to Ansys (ANSYS, Inc.) input file containing nodal coordinates and element conectivity.

Engineering simulation software Ansys (ANSYS, Inc.) was used for the numerical analysis. The simulation was assumed to be quasistatic divided in 20 loadsteps. Loading force value and direction of downstair walking in each load step are depicted in Fig. 6. Surface-to-surface contact was defined between head and acetabular component. The head and inner hemishere of the cup was meshed by contact elements to model femoro-acetabular interface. The contact was used for more accurate force transmission to the pelvis. Friction between the femoral head and the acetabular cup was neglected. Material properties are described in Tab. 1. All materials were considered as isotropic linear elastic.

Trabecular bone material properties were obtained by ultrasonic measurement, properties of the stainless steel, UHMWPE (ISO 5834 -2) and the bone-cement Palacos $\mathrm{R}$ (Heraeus Medical $\mathrm{GmbH}$ ) were provided by the manufacturers. 


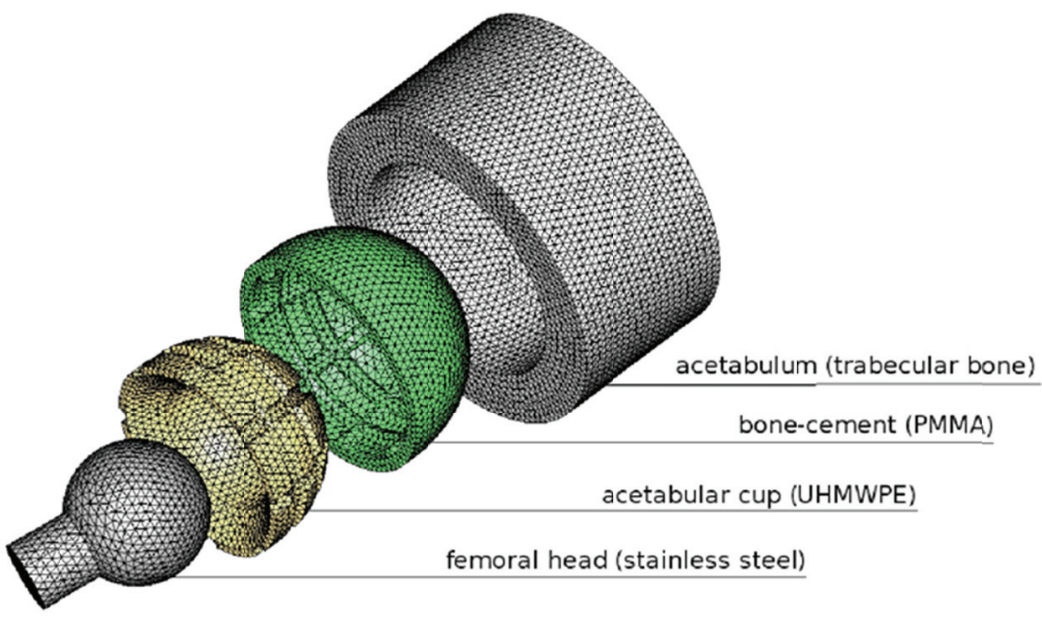

Figure 5: 10-node tetrahedral elements mesh.

\begin{tabular}{lcc}
\hline Material & Young's modulus [GPa] & Poisson's ratio \\
Stainless steel & 220 & 0.30 \\
UHMWPE (ISO 5834 -2) & 0.69 & 0.46 \\
Bone cement & 2 & 0.38 \\
Trabecular bone & 0.850 & 0.25 \\
\hline
\end{tabular}

Table 1: Material properties used in the FE model.

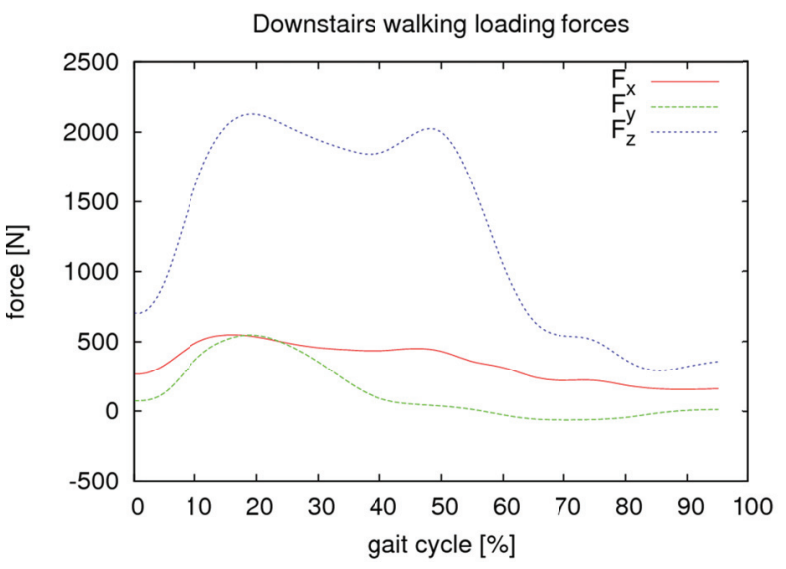

Figure 6: Loading force used in the simulation.

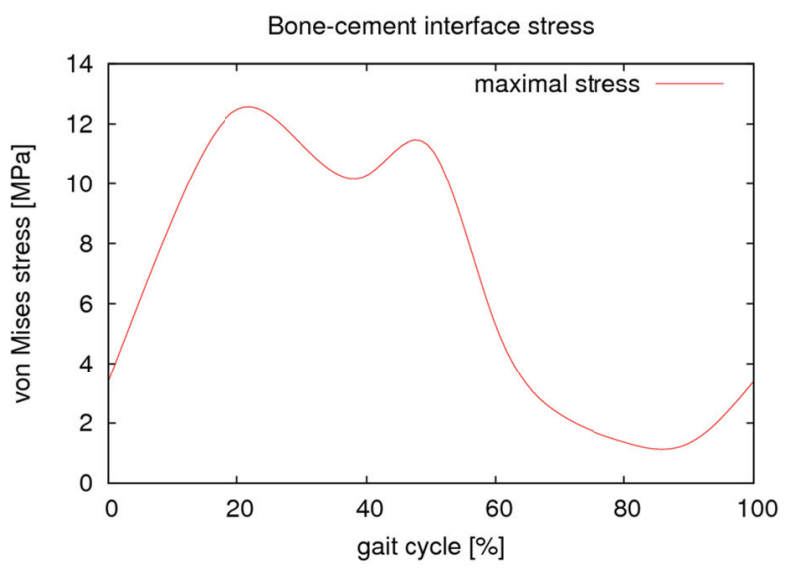

Figure 7: Resulting stress in the bone-cement interface.

The stress field distribution in the bone-cement interface were observed to locate the regions of potential damage. Maximum stress value equal to $12.48 \mathrm{MPa}$ was reached at $20 \%$ of the gait cycle. The stress fields on the bone-cement interface are shown in Fig. 8. The places of maximal value of von Mises stress were in the acetabular labrum for all load steps considered.

\section{Microtomography imaging}

To observe the process of damage in the acetabular region during the loading microfocus Computed Tomography $(\mu \mathrm{CT})$ imaging using a custom $\mu$ CT device shown in Fig. 9 was performed. Manipulation with the X-ray source, the table with the specimen and the detector were provided by stepper motors using a USB interface, controlled by Pixelman [8] software plug-in. 


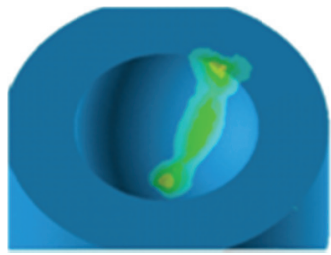

(a) $0 \%$ of gait cycle

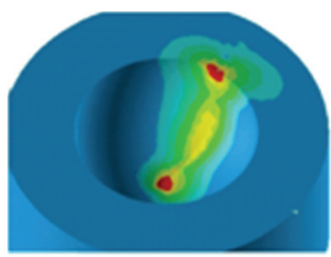

(e) $20 \%$ of gait cycle

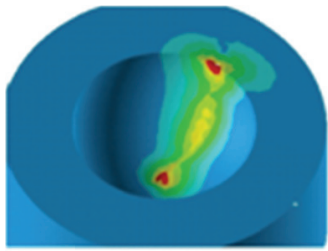

(i) $40 \%$ of gait cycle

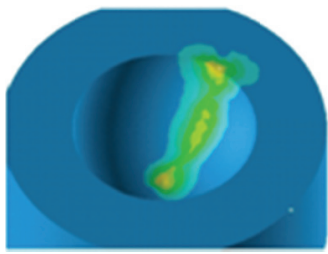

(m) $60 \%$ of gait cycle

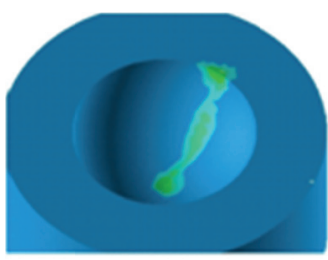

(q) $80 \%$ of gait cycle

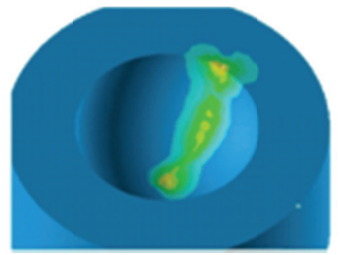

(b) $5 \%$ of gait cycle

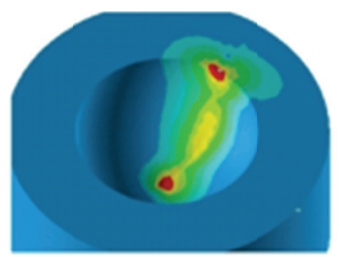

(f) $25 \%$ of gait cycle

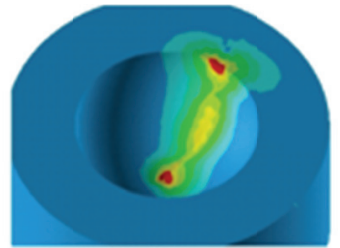

(j) $45 \%$ of gait cycle

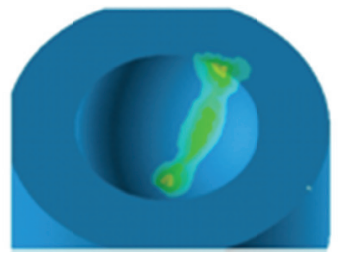

(n) $65 \%$ of gait cycle

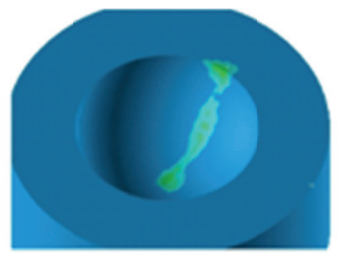

(r) $85 \%$ of gait cycle

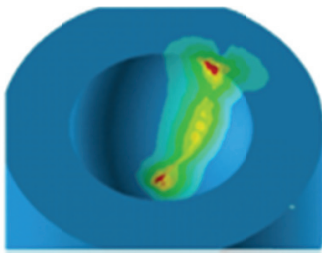

(c) $10 \%$ of gait cycle

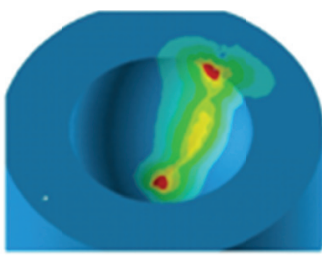

(g) $30 \%$ of gait cycle

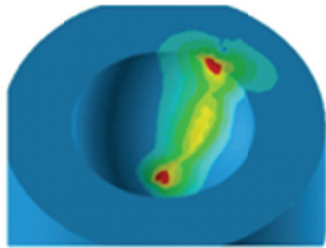

(k) $50 \%$ of gait cycle

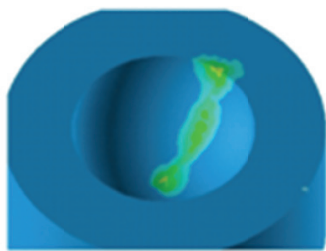

(o) $70 \%$ of gait cycle

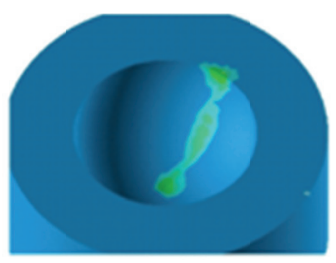

(s) $90 \%$ of gait cycle

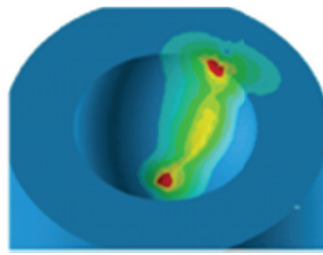

(d) $15 \%$ of gait cycle

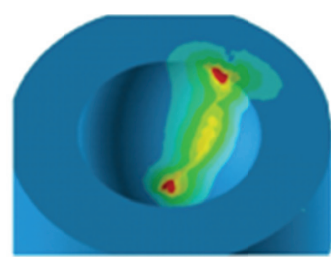

(h) $35 \%$ of gait cycle

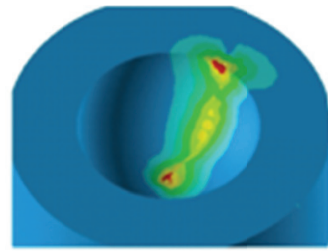

(l) $55 \%$ of gait cycle

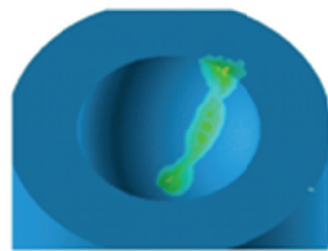

(p) $75 \%$ of gait cycle

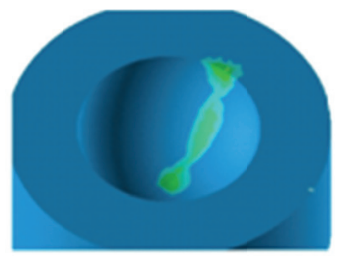

(t) $95 \%$ of gait cycle

Figure 8: von Mises stress distribution in the bone-cement interface during the normal walking cycle.

Microfocus X-ray source L8601-01 (Hamamatsu Photonics K.K.) with wolfram anode was used. For this purpose a flat panel detector (FPD) C7942CA-22 (Hamamatsu Photonics K.K.) with thin-film transistor (TFT) construction was used. Signal detected at each pixel of the detector was integrated to the memory. Physical dimensions of the detector are $120 \times$ $120 \mathrm{~mm}$ with maximal resolution of $4 \mathrm{Mpx}$. The pelvic bone was fixed in the iliac area onto a rotary table to leave the acetabular region exposed for scanning. Scanning sequence consisted of 360 scans with $1^{\circ}$ step increment. Acquisition was performed using 10 times $0.5 \mathrm{~s}$, voltage $80 \mathrm{kV}$ and current $125 \mu \mathrm{A}$. 
$\mu \mathrm{CT}$ provides detailed information about the internal structure. According to the density and composition of the specimen a fraction of the X-ray beam is absorbed. The X-rays that pass through are then captured and represented by output emission intensity I on detector. Relationship between input and output intensity of monochromatic X-ray is given by Beer-Lambert law:

$$
\frac{I}{I_{0}}=e^{-\mu(E) d}
$$

where $I_{0}$ is the value of input emission emitted by the X-ray source, $d$ is the thickness of the specimen and $\mu(E)$ is the linear attenuation coefficient:

$$
\mu=\rho \lambda^{3} Z^{4}
$$

where $\varrho$ is the density of absorbent material, Z material proton number and $\lambda \mathrm{X}$-rays wavelength.

Radiograph quality is mainly given by the number of photons contributing to the image. This number is proportional to the source power and the acquisition time. With decreasing number of photons the proportion of noise is increasing. Calibration measurements have been performed for the noise reduction. Flat field correction (scanning without specimen) for inhomogeneity reduction across the chip, dark current correction (scanning with disconnected source) to avoid the radiation background and beam hardening correction to get absorbing characteristics [9, 10], were performed. The set of corrected radiograms (3D radiation distribution in the 2D plane detector) were used for reconstruction of the scanned object. For the reconstruction Filtered Backprojection (FBP) was used.

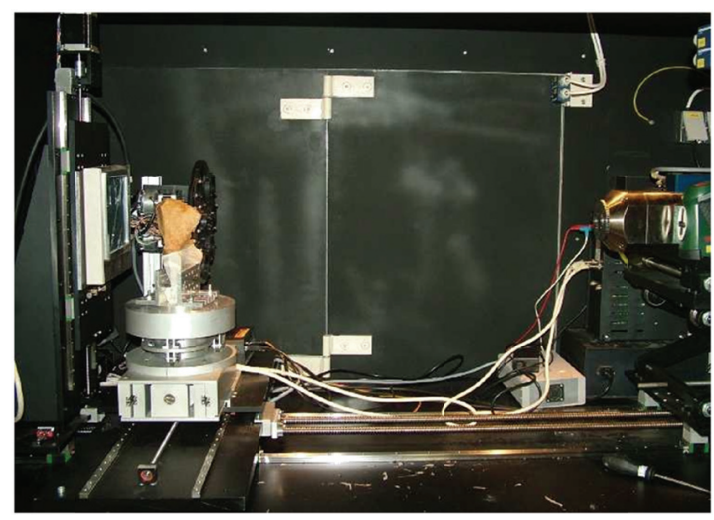

Figure 9: Microtomography device; pelvic bone sample on the left, X-ray source on the right.
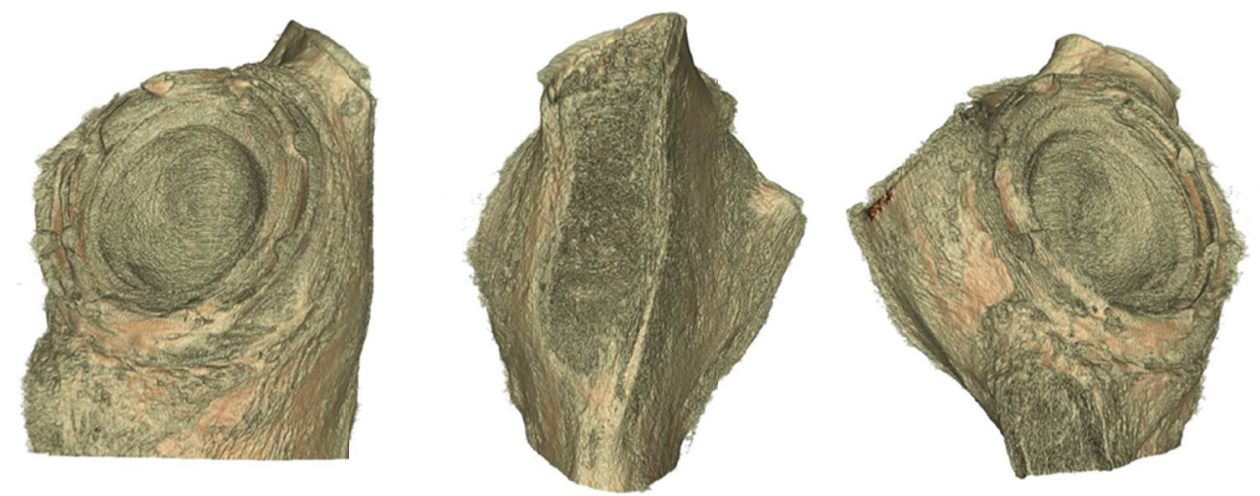

Figure 10: Reconstructed acetabulum from different views.

Projections obtained by $\mu \mathrm{CT}$ imaging were visualized using $\mu$ CTvis\&modeller software [12] based on open source Visualization Toolkit (VTK) libraries. The bone-cement interface before cyclic loading is shown in Fig. 11a, the damaged 
one is shown in Fig. 11b. Comparing Fig. 11a and Fig. 11b the direction of crack propagation is clearly visible. The location is in accord with the results obtained from FE analysis - stress field with its maximum in region of acetabular labrum.

\section{RESULTS}

$\mathrm{H}$

igh resolution models based on a sequence of 1100 slices with resolution $2368 \times 2240$ px and 16 bit color depth were developed. Visualisation of the pelvis with implantated acetabular component before mechanical testing is depicted in Fig. 10. The significant damage and direction of crack propagation in inner structure is observable in the segment of the model of the loaded pelvis. A place with initialized crack was found at the bone-cement interface. In accordance with the results obtained by the $\mathrm{FE}$ analysis - stress field with its maximum in region of acetabular labrum - the crack is observable in the same region (shown in Fig. 11).

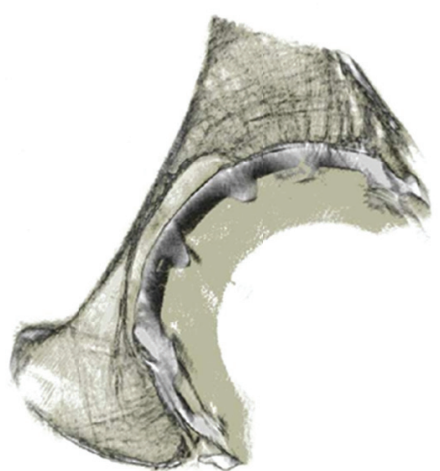

(a) intact

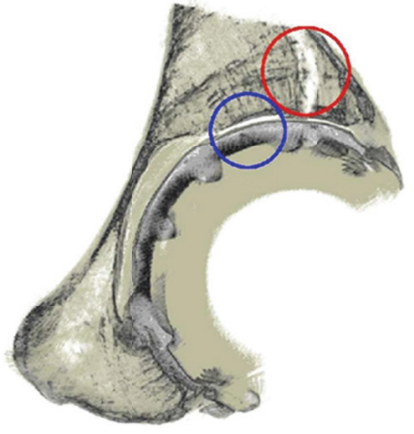

(b) damaged

Figure 11: Reconstructed slices of acetabulum; crack in region of acetabular labrum (red circle); debonding of bone-cement interface (blue circle).

\section{CONCLUSIONS}

his study shows possibilities of modern X-ray imaging techniques. Use of high resolution flat panel detector of large physical dimensions and micro-focus source allows for reconstruction of full-scale micro-structural models. These models are suitable for investigation of structural changes caused by osteoporosis as well as for research of trabeculae damage and total hip joint replacement behaviour. A failure in cemented acetabular implant - debonding, crumb ling and smeared cracks - has been found to be at the bone-cement interface, based on a new hip simulator study. Migration, motions and consecutive loosening of the implant can be observed by this method. The failure mechanism is consistent with clinically observed degradation process.

\section{ACKNOWLEDGEMENT}

he research has been supported by Grant Agency of the Czech Technical University in Prague (grant No. SGS10/218/OHK2/2T/16), by the Grant Agency of the Czech Republic (grant No. P105/10/2305), by research plan of the Ministry of Education, Youth and Sports MSM6840770043 and research plan of the Academy of Sciences of the Czech Republic AV0Z20710524.

\section{REFERENCES}

[1] G. Bergmann, G. Deuretzbacher, M. Heller, F. Graichen, A. Rohlmann, J. Strauss, G. Duda, J. of Biomechanics, 34(7) (2001) 859 . 
[2] N.P. Zant, C.K.Y Wong, J. Tong, Int. J. of Fatigue, 29(7) (2007) 1245.

[3] J. Tong, N.P. Zant, J.-Y. Wang, P. Heaton-Adegbile, J.G. Hussell, Int. J. of Fatigue, 30(8) (2008) 1366.

[4] Y.H. An, R.A. Draughn, Mechanical Testing of Bone and the Bone-Implant Interface, CRC Press (2000).

[5] D. Kytyr, J. Valach, O. Jirousek, D. Frankeova, J. Jira, In: $8^{\text {th }}$ YSESM Proceedings, Scientific Society of Mechanical Engineers, (2009).

[6] J.Rho, Medical and Biological Engineering and Computing, 36 (1998) 57.

[7] F. G. R. de Oliveira, Building and Environment, 40(2) (2005) 297.

[8] T. Holy, J. Jakubek, Pixelman software package - data acquisition and control of semiconductor pixel device Medipix, (2007).

[9] J. Jakubek, A. Cejnarova, T. Holy, S. Pospisil, J. Uher, Z. Vykydal, Nuclear Instruments and Methods in Physics Research, 576(1) (2007) 223.

[10] D. Vavrik, J. Jakubek, Nuclear Instruments and Methods in Physics Research Section A: Accelerators, Spectrometers, Detectors and Associated Equipment, 607 (2009) 212.

[11] D. Vavrik, T. Holy, J. Jakubek, M. Jakubek, J. Valach, Czechoslovak Journal of Physics, 58(1) (2008) 58.

[12] P. Zlamal, O. Jirousek: $\mu$ CTmodeller - creating solid and Finite Element models from microCT \& $\mu$ CTvis visualization of microfocus Computed Tomography data, (2009). 